PAPER

\title{
Association between apolipoprotein E e4 allele and arteriosclerosis, cerebral amyloid angiopathy, and cerebral white matter damage in Alzheimer's disease
}

\author{
J Tian, J Shi, K Bailey, C L Lendon, S M Pickering-Brown, D M A Mann
}

J Neurol Neurosurg Psychiatry 2004;75:696-699. doi: 10.1136/jnnp.2003.012096

See end of article for authors' affiliations

.....................

Correspondence to: Professor D M A Mann, Clinical Neuroscience Research Group, Greater Manchester Neurosciences Centre, Hope Hospital, Stott Lane, Salford, M6 8HD, UK; david.mann@ man.ac.uk

Received 3 February 2003 In revised form

19 August 2003

Accepted 23 August 2003

\begin{abstract}
Objective: To investigate the association between white matter damage, as evidenced by myelin loss (ML), the extent of cerebral amyloid angiopathy (CAA), or arteriosclerosis (Art), and apolipoprotein E (ApoE) e4 allele in Alzheimer's disease (AD), in order to understand the causes of damage to white matter in $A D$ and its contribution to the pathogenesis of the disorder.

Materials and methods: Brain tissues were obtained from 94 patients with AD confirmed by autopsy. ApoE genotyping was performed by PCR on DNA extracted from frontal cortex or cerebellum. CAA and Art were assessed on Weigert's haematoxylin and eosin stained sections in frontal, temporal, parietal, and occipital cortices; the extent of ML was scored on Luxol fast blue stained sections of these regions.

Results: The ApoE e4 allele frequency in the 61 patients with ML was not significantly different from that in the 33 patients without ML, nor did this differ in the 84 patients with Art from that in the 10 patients without Art. There were no significant differences in the proportions of patients with genotypes containing 0,1 , or 2 ApoE e4 alleles in the presence or absence of ML or Art. The mean ML, Art, or CAA scores within each region, and the total scores summed across all four brain regions, did not differ between patients with 0,1 , or $2 \mathrm{ApoE}$ e4 alleles. However, the mean ML severity score in the occipital cortex was significantly greater than that in the frontal or temporal cortices in patients with 1 or 2 ApoE e4 alleles. The severity of CAA in the occipital cortex was significantly higher than that in other areas of cortex in patients with 0 or $2 \mathrm{ApoE}$ e4 alleles. The mean Art score in the occipital cortex was greater than that in the temporal cortex in patients with two ApoE e4 alleles and was higher than that in the frontal cortex in patients with one ApoE e4 allele.

Conclusions: The likelihood of patients with AD suffering from CAA, Art, or ML is not influenced by ApoE e4 allele, nor is the overall burden of these pathological changes in the brain. However, the distribution of ML, CAA, and Art within the brain is at least partly influenced by genotype and dosage of ApoE e4 allele, with the occipital cortex being more severely affected by all of these pathological changes in e4 allele bearers, particularly when two ApoE e4 alleles are present.
\end{abstract}

hypoperfusion caused by fibrohyalinosis of the medullary arteries. $^{13}{ }^{14}$

Although the apolipoprotein E (ApoE) e4 allele may be associated with a higher burden of $A \beta$ (especially $A \beta_{40}{ }^{15}{ }^{16}$ but see Chalmers et $a l^{7}$ ), NFT, ${ }^{17}{ }^{18}$ and phosphorylated tau ${ }^{19}$ in the brain parenchyma, the influence of the ApoE genotype on the extent of vascular pathology and white matter changes in $\mathrm{AD}$ is controversial. In one study, ${ }^{20}$ patients with the ApoE e3/e4 and e4/e4 genotypes had more extensive white matter lesions than patients with the e3/e3 genotype. Skoog et $a^{21}$ reported that risk of dementia in very old people is associated with white matter loss and the ApoE e4 allele (but not each alone), suggesting an interaction between these two factors in the causation of dementia. However, in other reports, ${ }^{22-24}$ no associations with the presence or degree of white matter loss have been found. The extent of CAA has been linked in some studies to possession of the ApoE e4 allele, ${ }^{45} 7^{25-27}$ especially in patients with the e4/e4 genotype, ${ }^{67}$ but other workers $^{28}{ }^{29}$ have not found such an association. Variations in findings may in part be due to the small sample sizes often

Abbreviations: $A \beta$, amyloid $\beta$ protein; $A D$, Alzheimer's disease; $A p o$, apolipoprotein; Art, arteriosclerosis; BA, Brodmann area; CAA, cerebral amyloid angiopathy, LFB, Luxol fast blue; ML, myelin loss; MRI, magnetic resonance imaging; WHE, Weigert's haematoxylin and eosin. with $\mathrm{AD}^{10-12}$ and it is therefore also possible that white matter damage and loss in $\mathrm{AD}$ results from a chronic cerebral 
employed, often with few patients homozygous for the ApoE e4 allele, and the variety of assessment methods used.

In this study we have investigated whether the ApoE e4 allele may constitute a risk factor for cerebral white matter damage in $\mathrm{AD}$ in its own right, or through its interactions with either CAA or Art. For this, we investigated the presence and distribution of white matter damage (in terms of myelin loss; ML), CAA, and Art in four brain regions of a large cohort of 94 patients with $\mathrm{AD}$, and correlated these with possession of one or two copies of the ApoE e4 allele.

\section{MATERIALS AND METHODS}

All brain tissues used in this study had been obtained from patients in whom the diagnosis of $\mathrm{AD}$ had been confirmed at autopsy according to CERAD neuropathological criteria. ${ }^{30}$ Patients with diffuse Lewy bodies, large embolic infarcts, or primary vascular dementia in addition to $\mathrm{AD}$ lesions were excluded from the study. Ninety four patients (50 females: mean (SD) age at onset, 65.4 (10.3) years; age at death, 74.3 (10.1) years; duration of illness, 7.9 (2.9) years; 44 males: age at onset, 64.1(10.4) years; age at death, 73.1 (9.4) years; duration of illness, 8.1 (3.3) years) with clinical and neuropathological evidence of $\mathrm{AD}$ were investigated. There were no significant differences in age at onset, age at death, or duration of illness between males and females.

Formalin fixed blocks of frontal (Brodmann area (BA) 8/9), temporal (BA 21/22), occipital (BA 17/18), and parietal (BA 39/40) cortex were similarly and routinely embedded in paraffin wax, and consecutive sections, cut at a thickness of $6 \mu \mathrm{m}$, were mounted onto 3-aminopropyltriethoxysilane coated slides. Sections were stained by Weigert's haematoxylin and eosin (WHE) method to assess the severity of CAA and Art, and by Luxol fast blue (LFB) $)^{31}$ to assess the extent of ML as an index of white matter damage. The severity of CAA, the degree of Art, and the extent of ML were assessed semiquantitatively in each of the four cortical areas, as described elsewhere, ${ }^{32}$ and scores were summed across regions to provide a combined severity score for each patient. Our previous studies have shown that the severity of CAA can be detected as efficiently in WHE stained sections as in sections immunostained for $A \beta .^{32}$ LFB, however, is not a progressive stain, requiring differentiation in lithium carbonate for optimal histological demonstration of myelin. ${ }^{31}$ This could potentially induce some batch to batch variation in stained product. However, in this present study, LFB staining was quality controlled by including a known normal "control" case in each batch of sections from the AD cases in which the degree of myelin staining could be checked. Hence, it was possible to compare the degree of myelin staining in each of the $\mathrm{AD}$ cases on an equivalent basis. Moreover, even in those AD cases where ML was severe, there was residual well stained myelin in undamaged parts of the white matter in one or more of the sections from each case that could further act as an "internal standard" to check on the reproducibility of LFB staining compared with that in the control case.

ApoE genotyping was determined from DNA extracted from frozen samples of frontal cortex or cerebellum using PCR. $^{33}$

One way non-parametric analysis of variance (KruskalWallis, using SPSS software) was applied to categorical variables when comparing differences in pathology ratings across brain regions for bearers of 0,1 , or 2 ApoE e 4 alleles, or between bearers of 0,1 , or 2 ApoE e4 alleles for each brain region. Mann-Whitney $U$ test was used post hoc to identify significant differences between groups/regions. $\chi^{2}$ test was used to identify differences in frequency of affected cases within genotype groups. Two tailed $p$ values $\leqslant 0.05$ were considered statistically significant throughout all analyses.

\section{RESULTS}

\section{Myelin loss}

Myelin loss was found in 61 patients (65\%). The ApoE e4 allele frequency in these 61 cases with ML (50/122 alleles, 0.41 ) was not significantly different from that in the 33 cases without ML (23/66 alleles, 0.35), nor were there any significant differences in the percentages of patients with each ApoE genotype, or between the percentage of patients with 0,1 , or 2 e 4 alleles, in the presence or absence of ML (fig lA). The mean ML score within each region in the 61 patients with ML (table 1), and the total score summed across all four brain regions (fig 2A) did not differ according to ApoE genotype, or between patients with 0 , 1 , or 2 e 4 alleles. However, there were significant differences in the severity of ML scores between the four brain regions according to possession of ApoE e4 allele (table 1). Thus, the mean ML score in the occipital cortex in patients with 1 or 2 ApoE e4 alleles was higher $(p<0.05-0.01)$ than that in frontal and temporal cortex (and parietal cortex when 2 e4 alleles were present) whereas there were no such regional differences in patients with no e4 alleles) (table 1).

\section{CAA}

All 94 patients showed CAA to a greater or lesser extent, although not all brain areas were always or equally affected in every patient. The presence of CAA was therefore independent of ApoE genotype. The mean CAA score within each region and the mean total CAA score summed across all four brain regions (fig $2 \mathrm{~B}$ ) did not differ between patients with 0,1 , or 2 ApoE e4 alleles. However, the severity of CAA in the occipital cortex in cases with 0 or 2 APO e 4 alleles was significantly higher $(\mathrm{p}<0.05-0.01)$ than that in the other regions of cortex, but there were no such regional differences in patients with 1 APO e4 allele (table 1).

\section{Arteriosclerosis}

Arteriosclerosis was detected to some degree in 84 patients (89\%). The ApoE e4 allele frequency in the 84 cases with Art (67/168 alleles, 0.40$)$ was not significantly different from that in the 10 cases without Art (9/20 alleles, 0.45), nor were there any significant differences in the percentages of patients with 0 , 1, or 2 e4 ApoE alleles in the presence or absence of Art (fig 1B). The mean Art score within each region (table 1) and the mean total Art score summed across all four brain regions
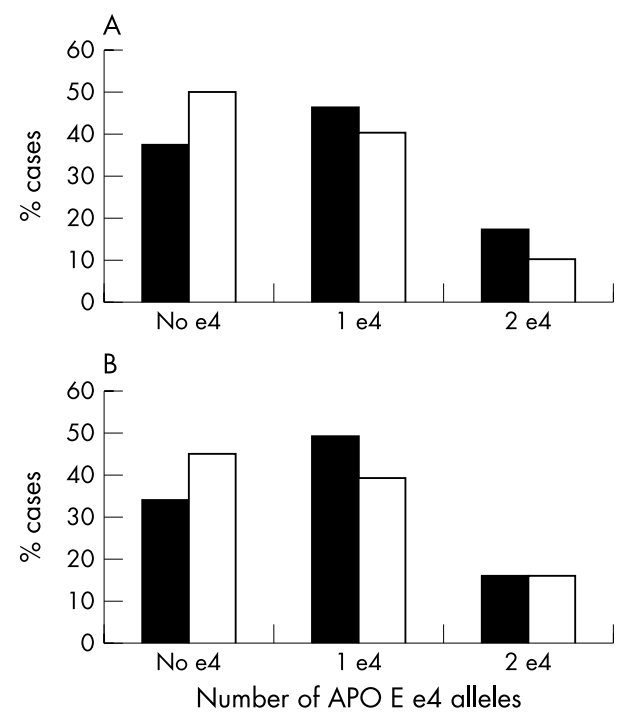

Figure 1 Percentage of cases with (black bars) and without (white bars) $\mathrm{ML}(\mathrm{A})$ or Art (B) according to the presence of 0,1 , or $2 \mathrm{ApoE}$ e4 (e4) alleles. 


\begin{tabular}{|c|c|c|c|c|c|c|}
\hline & e4 alleles & $\begin{array}{l}\text { Number of } \\
\text { cases }\end{array}$ & Frontal & Temporal & Parietal & Occipital \\
\hline \multirow[t]{3}{*}{ ML } & None & 21 & $0.9(1.0)$ & $0.7(0.9)$ & $0.8(0.9)$ & $1.0(0.7)$ \\
\hline & 1 & 30 & $0.3(0.7)^{* *}$ & $0.4(0.7)^{* *}$ & $1.1(1.0)$ & $1.2(0.9)$ \\
\hline & 2 & 10 & $0.3(0.7)^{* *}$ & $0.6(1.1)^{*}$ & $0.6(0.7)^{*}$ & $1.4(0.8)$ \\
\hline \multirow[t]{3}{*}{ CAA } & None & 36 & $1.5(0.8)^{* *}$ & $1.6(0.7)^{*}$ & $1.7(0.9)$ & $2.5(0.8)$ \\
\hline & 1 & 43 & $1.7(0.8)$ & $1.6(0.8)$ & $1.7(0.9)$ & $2.0(0.9)$ \\
\hline & 2 & 15 & $1.9(0.8)^{*}$ & $1.8(0.6)^{* *}$ & $1.9(1.1)$ & $2.5(0.8)$ \\
\hline \multirow[t]{3}{*}{ Art } & None & 31 & $2.6(2.2)$ & $2.2(2.5)$ & $2.9(2.6)$ & $3.3(2.3)$ \\
\hline & 1 & 39 & $1.9(2.2)^{* *}$ & $2.3(1.8)$ & $3.0(2.4)$ & $3.2(2.6)$ \\
\hline & 2 & 41 & $2.8(2.6)$ & $1.9(2.2)^{*}$ & $2.7(2.7)$ & $4.5(2.5)$ \\
\hline
\end{tabular}

(fig 2C) did not differ between patients with 0, 1, or 2 ApoE e4 alleles. The mean Art score in the occipital cortex was higher $(p<0.05)$ than that in the temporal cortex in patients with 2 ApoE e4 alleles. In patients with one ApoE e4 allele, mean Art score in the occipital cortex was also higher $(\mathrm{p}<0.05)$ than that in the frontal cortex (table 1$)$.

\section{DISCUSSION}

Present data suggest that the likelihood of patients with $\mathrm{AD}$ suffering from CAA, Art, or ML is not influenced by ApoE genotype, nor is the overall burden of these pathological changes in the brain so influenced. However, the actual distribution of ML, CAA and Art within the brain with $\mathrm{AD}$ may be at least partly influenced by genotype and dosage of ApoE e4 allele. Hence, the occipital cortex seems to be preferentially affected by all three of the pathological
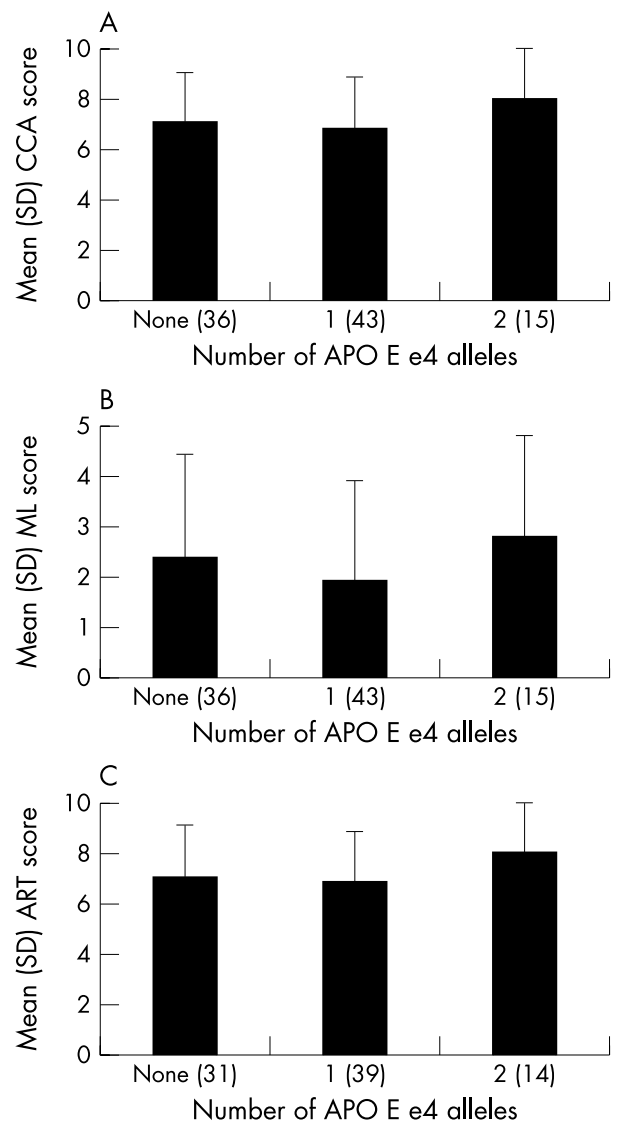

Figure 2 Mean (+SD) severity scores for ML (A), CAA (B) and Art (C) according to the presence of 0,1 , or $2 \mathrm{ApoE} \mathrm{e} 4$ (e4) alleles. Number of cases is given in parenthesis. changes, compared with other brain regions, particularly so in the presence of the ApoE e4/e4 genotype.

The influence of ApoE genotype on the extent of white matter damage and loss in $\mathrm{AD}$ is unclear. In MRI studies, the possession of the ApoE e4 allele has been linked in some studies $^{2021}$ to white matter lesions but in other MRI based studies no correlation between white matter scores and ApoE genotype was found..$^{22-24}$ Autopsy based studies are few, and often based on small cohorts, but these too suggest no association between the ApoE e4 allele and white matter loss. $^{34} 35$ Present data, based on 94 autopsy assessed AD patients, indicate that while the ApoE genotype is not associated with the likelihood of ML occurring in the brain in $\mathrm{AD}$, it may influence the location and severity of ML, with the occipital cortex being more frequently and more severely affected, compared with other brain regions, in patients with ApoE e3/e4 and e4/e4 genotypes. Further studies are needed to clarify the association between loss and damage to white matter and the ApoE genotype.

The prevalence of CAA within the brain in patients with $\mathrm{AD}$ has been estimated to vary from about 81 to $98 \%$ of cases. $^{4-7}$ As previously reported, ${ }^{45736}$ most patients with AD in this present study were overall moderately or severely affected by CAA, with the occipital cortex being affected more than the frontal, temporal, and parietal cortices. Possession of the ApoE e4 allele in $\mathrm{AD}$ has been claimed to correlate with the extent of CAA, ${ }^{425-27}$ especially in patients bearing ApoE e4/e4 genotype. ${ }^{57}$ However, not all investigators have confirmed such an association. ${ }^{28}{ }^{29}$ In the present study we were unable to show any effect of possession of ApoE e4 allele on either the likelihood of CAA being present in the brain, or the overall severity of CAA. It is possible that different ways of histologically demonstrating CAA and grading the degree of severity between the various studies may have led to such discrepancies of finding. Although in the present report, CAA was assessed on WHE stained sections, we have found a similar lack of effect of ApoE e4 allele in a subset of the 94 patients examined here using $\beta$-amyloid immunostaining ( $\mathrm{J}$ Tian, unpublished data). Most previous studies on CAA in $\mathrm{AD}^{4} 7^{25-29}$ have not studied the effects of ApoE genotype on the distribution and severity of CAA in the brain. Our present data suggest that the distribution of CAA in $\mathrm{AD}$ may be, at least partly, influenced by genotype and dosage of ApoE e4 allele, with the occipital cortex being most frequently and most severely damaged by CAA in those patients carrying ApoE e4/e4 genotype. This predilection for occipital cortex to be affected severely in cases with ApoE e4/e4 genotype was also noted by Zarow et al. ${ }^{5}$ Hence, the distribution and severity of CAA within the brain in a patient with $\mathrm{AD}$ may be "driven" by the ApoE e4 allele, although variations in overall severity of CAA between patients with $\mathrm{AD}$ cannot be explained by the ApoE genotype alone. 
In the present study the distribution (but not the overall severity) of Art was also related to the ApoE e4 allele, with the occipital cortex again being affected more than any other brain region, especially in patients with the ApoE e4/e4 genotype. Therefore, as with CAA, the distribution of Art in the brain with $\mathrm{AD}$ may likewise be partly influenced by the ApoE e4 allele, which may act as a shared risk factor, explaining (in part) the association between the extent of CAA and Art in the brain in $\mathrm{AD}^{31}$ although it is likely that other genetic or non-genetic factors may also be responsible.

It is interesting that the occipital lobe has more severe pathology for all three measures, ML, CAA, and Art, but only in ApoE e4 allele bearers. Does this mean that there is something intrinsically different about the occipital lobe, or the posterior cerebral circulation, compared with the rest of the brain, which makes this part of the brain especially vulnerable to vascular pathology? If so, the ApoE e4 protein may exacerbate such inherent vulnerability, perhaps through impaired tissue maintenance and response to damage.

\section{Authors' affiliations}

J Tian, J Shi, K Bailey, D M A Mann, Clinical Neuroscience Research Group, University of Manchester, Hope Hospital, Salford, Manchester M6 8HD, UK

J Tian, J Shi, Department of Care of the Elderly, Dongzhimen Hospital, Beijing University of Chinese Medicine, Beijing 100700, China C L Lendon, Department of Psychiatry, University of Birmingham, Birmingham B15 2QZ, UK

S M Pickering-Brown, Department of Old Age Psychiatry, Institute of Psychiatry, London SE5 8AF, UK

Competing interests: none declared

\section{REFERENCES}

1 Scheltens PH, Barkhof F, Valk J, et al. White matter lesions in magnetic resonance imaging in clinically diagnosed Alzheimer's disease. Brain 1992; 115:735-48.

2 Pantoni L, Garcia JH, Gutierrez JA. Cerebral white matter is highly vulnerable to ischaemia. Stroke 1996;27:1641-6.

3 Leys D, Scheltens $\mathrm{P}$, Steinling M. White matter lesions on $\mathrm{CT}$ in Alzheimer patients. Acta Neurol Scand 1991;84:264-5.

4 Premkumar DR, Cohen DL, Hedera P, et al. Apolipoprotein E $\epsilon 4$ alleles in cerebral amyloid angiopathy and cerebrovascular pathology associated with Alzheimer's disease. Am J Pathol 1996;148:2083-95.

5 Zarow C, Zaias B, Lyness SA, et al. Cerebral amyloid angiopathy in Alzheimer disease is associated with Apolipoprotein E4 and cortical neuron loss. Alzheimer Dis Assoc Disord 1999;13:1-8.

6 Jellinger K. Alzheimer disease and cerebrovascular pathology-an update. J Neural Transm 2002;109:813-36.

7 Chalmers K, Wilcock GK, Love S. APO E $\in 4$ influences the pathological phenotype of Alzheimer's disease by favouring cerebrovascular over parenchymal accumulation of $A \beta$ protein. Neuropathol Appl Neurobiol 2003;29:231-8.

8 Gray F, Dubas F, Roullet E, et al. Leukoencephalopathy in diffuse haemorrhagic cerebral amyloid angiopathy. Ann Neurol 1985;18:54-9.

9 Vinters HV, Ellis WG, Zarow C, et al. Neuropathologic substrates of ischaemic vascular dementia. J Neuropathol Exp Neurol 2000;59:931-45.

10 Brun A, Englund E. White matter disorders in dementia of the Alzheimer's type: biochemical and neuropathological correlates. Ann Neurol 1986;19:253-62
11 Ferrer I, Bella R, Marti SE, et al. Arteriosclerotic leucoencephalopathy in the elderly and its relation to white matter lesions in Binswanger's disease, multiinfarct encephalopathy and Alzheimer's disease. J Neurol Sci 1990:98:37-50

12 Tominoto $\mathrm{H}$, Akiguchi I, Akiyama $\mathrm{H}$, et al. Vascular changes in white matter lesions of Alzheimer's disease. Acta Neuropathol 1999;97:629-34.

13 Furuta A, Ishii N, Nishihara Y, et al. Medullary arteries in aging and dementia. Stroke 1991;22:442-6.

14 Pantoni L, Garcia JH. Pathogenesis of leukoaraiosis. A review. Stroke 1997:28:652-9.

15 Gearing M, Mori H, Mirra SS. A $\beta$ peptide length and apolipoprotein E genotype in Alzheimer's disease. Ann Neurol 1996;39:395-9.

16 Mann DMA, Iwatsubo T, Pickering-Brown SM, et al. Preferential deposition of amyloid $\beta$ protein $(A \beta)$ in the form $A \beta_{40}$ in Alzheimer's disease is associated with a gene dosage effect of the apolipoprotein E E4 allele. Neurosci Lett 1997;221:81-4

17 Nagy Z, Esiri MM, Jobst KA, et al. Influence of the apolipoprotein E genotype on amyloid deposition and neurofibrillary tangle formation in Alzheimer's disease. Neuroscience 1995;69:757-61.

$18 \mathrm{Ohm}$ TG, Kirca M, Bohl J, et al. Apolipoprotein E polymorphism influences not only cerebral senile plaque load but also Alzheimer-type neurofibrillary tangle formation. Neuroscience 1995;66:583-7.

19 Thaker U, McDonagh AM, Iwatsubo T, et al. Tau load is associated with apolipoprotein $E$ genotype and the amount of amyloid $\beta$ protein, $A \beta_{40}$, in sporadic and familial Alzheimer's disease. Neuropathol Appl Neurobiol 2003;29:35-44.

20 Bronge $\mathrm{L}$, Fernaeus SE, Blomberg $M$, et al. White matter lesions in Alzheimer patients are influenced by apolipoprotein E genotype. Dement Geriatr Cogn Disord 1999;10:89-96.

21 Skoog I, Hesse C, Aervarsson O, et al. A population study of apo $E$ genotype at the age of 85: relation to dementia, cerebrovascular disease, and mortality. J Neurol Neurosurg Psychiatry 1998;64:37-43.

22 Barber R, Gholkar A, Scheltens P, et al. Apolipoprotein E $\in 4$ allele, temporal lobe atrophy, and white matter lesions in late-life dementias. Arch Neurol 1999;56:961-5.

23 Doody RS, Azher SN, Haykal HA, et al. Does APO $€ 4$ correlate with MRI changes in Alzheimer's disease? J Neurol Neurosurg Psychiatry 2000;69:668-71

24 Sawada H, Udaka F, Izumi Y, et al. Cerebral white matter lesions are not associated with apoE genotype but with age and female sex in Alzheimer's disease. J Neurol Neurosurg Psychiatry 2000;68:653-6.

25 Greenberg SM, Rebeck GW, Vonsattel JPV, et al. Apolipoprotein E $\epsilon 4$ and cerebral haemorrhage associated with amyloid angiopathy. Ann Neurol 1995;30:637-49.

26 Greenberg SM, Briggs ME, Hyman BT, et al. Apolipoprotein E $\epsilon 4$ allele is associated with the presence and earlier onset of haemorrhage in cerebral amyloid angiopathy. Stroke 1996;26:1333-7.

27 Olichney JM, Hansen LA, Galasko D, et al. The apolipoprotein $\epsilon 4$ allele is associated with increased neuritic plaques and cerebral amyloid angiopathy in Alzheimer's disease and Lewy body variant. Neurology 1996:47:190-6.

28 Heinonen $\mathrm{O}$, Lehtovirta $M$, Soininen $\mathrm{H}$, et al. Alzheimer pathology of patients carrying Apolipoptoein E $\in 4$ allele. Neurobiol Ageing 1995;16:505-13.

29 Itoh Y, Yamada M, Suematsu N, et al. Influence of Apolipoprotein E genotype on cerebral amyloid angiopathy in the elderly. Stroke 1996;27:216-8.

30 Mirra SS, Heyman A, McKeel D, et al. The consortium to establish a registry for Alzheimer's disease. Part II. Standardization of the neuropathologic assessment of Alzheimer's disease. Neurology 1991;41:479-86.

31 Bancroft JD, Stevens A. Theory and practice of histological techniques. London: Churchill Livingstone, 1996.

32 Tian J, Shi J, Bailey K, et al. Relationships between arteriosclerosis and cerebral amyloid angiopathy and myelin loss from the cerebral cortical white matter in Alzheimer's disease. Neuropathol Appl Neurobiol 2004;30:46-56.

33 Wenham PR, Price WH, Blundell C. Apolipoprotein E genotyping by one-stage PCR. Lancet 1991;337:1158-9.

34 Goulding JMR, Signorin DF, Chatterjee S, et al. Inverse relation between Braak stage and cerebrovascular pathology in Alzheimer's predominant dementia. J Neurol Neurosurg Psychiatry 1999;69:654-7.

35 Jellinger K. Inverse relation between Braak stage and cerebrovascular pathology in Alzheimer predominant dementia. J Neurol Neurosurg Psychiatry 2000;68:799-800.

36 Pfeiffer LA, White LR, Ross GW, et al. Cerebral amyloid angiopathy and cognitive function: the HAAS autopsy study. Neurology 2002;58:1629-34. 